\title{
CONDIÇÕES CRÔNICAS EM DOCENTES DO ENSINO SUPERIOR: REVISÃO INTEGRATIVA
}

CHRONIC CONDITIONS IN POSTSECONDARY TEACHERS: INTEGRATIVE REVIEW

CONDICIONES CRÓNICAS EN PROFESORES DE EDUCACIÓN SUPERIOR: REVISIÓN INTEGRATIVA

Karla Beatriz Barros de Almeida ${ }^{1}$

Juliana Soares Androlage 2

Reni Barsaglini ${ }^{3}$

Palavras-chave:

Docentes; Doenças crônicas;

Trabalho.

Keywords:

Faculty; Chronic Diseases; Work.

Palabras clave:

Docentes; Enfermedades crónicas;

Trabajo.

Submetido:

$14 / 03 / 2021$

Aprovado:

$07 / 06 / 2021$

Autor(a) para Correspondência:

Karla Beatriz Barros de Almeida E-mail: karla.almeida@ifmt.edu.br

\section{RESUMO}

Objetivou-se identificar e descrever as características dos estudos científicos nacionais e internacionais sobre cronicidade em docentes universitários. Realizou-se revisão integrativa da literatura abrangendo a Biblioteca Virtual de Saúde, SciELO, CINAHL e Web of Science. Após criteriosa seleção, chegouse a 62 estudos para análise. Dentre os resultados, estudos brasileiros predominaram e a condição crônica mais abordada foi o estresse. As condições crônicas vivenciadas por docentes universitários foram ressaltadas nas discussões sobre: associação entre trabalho docente e cronicidade; questões de gênero, enfocando consequências da inserção da mulher no trabalho; intensificação do trabalho docente; correlação entre hábitos de vida e saúde; contexto pandêmico da Covid-19; e suporte institucional à saúde dos docentes universitários. Em conclusão, incentiva-se a ampliação: do desenvolvimento da temática no âmbito de estudos qualitativos; da realização de estudos sobre cronicidade por deficiencias nos docentes universitários; do estudo das implicações da pandemia pelo novo coronavírus considerando nessa população situações cronificadas que podem ser agravadas.

\footnotetext{
1. Enfermeira. Doutoranda em Enfermagem pela Universidade Federal de Mato Grosso (UFMT). Enfermeira do trabalho do Instituto Federal de Educação, Ciência e Tecnologia de Mato Grosso (IFMT). E-mail: karla.almeida@ ifmt.edu.br. ORCID: https://orcid.org/0000-0003-3874-4809

2. Enfermeira. Doutoranda em Enfermagem pela Universidade Federal de Mato Grosso (UFMT).E-mail: juhsoaress@ yahoo.com.br. ORCID: https://orcid.org/0000-0002-9993-5951

3. Pedagoga sanitarista. Doutora em Saúde Coletiva. Docente do Instituto de Saúde Coletiva da Universidade Federal de Mato Grosso (ISC-UFMT). E-mail: barsaglinireni@gmail.com. 0RCID: https://orcid.org/0000-0002-8903$\underline{2695}$
}

Cert. de Redação Científica: Central das Revisões. Edição de texto: Karina Maria M. Machado. Revisão de provas: Texto definitivo validado pelos(as) autores(as). 


\section{ABSTRACT}

The objective was to identify and describe the characteristics of national and international scientific studies on chronic conditions suffered by postsecondary teachers. An integrative literature review was carried out using the following databases: Biblioteca Virtual de Saúde, SciELO, CINAHL, and Web of Science. After careful selection, 62 studies were selected for analysis. Among the results, there was a predomination of Brazilian studies, and the chronic condition most frequently addressed was stress. The chronic conditions experienced by faculty were highlighted in discussions about the association between teaching and chronic conditions; gender issues, focusing on the consequences of women's insertion in work; intensification of the work teaching; correlation between lifestyle and health; Covid-19 pandemic context; institutional health support for postsecondary teachers. As a conclusion, we encourage the expansion of: thematic developments in the context of qualitative studies; studies on chronic conditions due to disabilities of faculty members; studies on the implications of the new coronavirus pandemic considering chronic conditions that can be aggravated in this population.

\section{RESUMEN}

Se objetivó identificar y describir las características de los estudios científicos nacionales e internacionales acerca de la cronicidad en los profesores universitarios. Se realizó una revisión integrativa de la literatura alcanzando la Biblioteca Virtual de Saúde, SciELO, CINAHL y Web of Science. Tras detallada selección, se llegó a 62 estudios para análisis. Entre los resultados, estudios brasileños predominaron y la condición crónica más abordada fue el estrés. Las condiciones crónicas vividas por los docentes universitarios fueron resaltadas en las discusiones sobre: asociación entre trabajo docente y cronicidad; cuestiones de género, focalizando consecuencias de la inserción de la mujer en el mundo laboral; intensificación del trabajo docente; correlación entre habitos de vida y salud; contexto pandémico de la Covid-19; asistencia institucional a la salud de los docentes universitarios. En suma, se incentiva la ampliación: del desarrollo de la temática en el ámbito de estudios cualitativos; de la realización de estudios sobre cronicidad por deficiencias en los docentes universitarios; del estudio de las implicaciones de la pandemia por el nuevo coronavirus considerando en esa población situaciones cronificadas que pueden agravarse.

\section{INTRODUÇÃO}

Condições crônicas são situações com caráter persistente, que abalam a saúde das pessoas e exigem ações reativas ou proativas, esporádicas ou contínuas, fragmentadas ou integradas, dos serviços de saúde e das pessoas que convivem com tais condições ${ }^{1}$. Esse conceito envolve doenças crônicas não transmissíveis, doenças infecciosas persistentes, condições relativas à maternidade e ao período perinatal; condições ligadas à manutenção da saúde por ciclos de vida; distúrbios mentais; deficiências físicas e estruturais; doenças metabólicas; doenças bucais não agudas e enfermidades (illnesses) ${ }^{1}$.

Diferentemente das doenças agudas, que interrompem temporariamente a vida no cotidiano, nas doenças crônicas se nota certa desestabilização com períodos de agudização e remissão, numa sucessão de dias bons e maus, bem como prolongada interrupção das rotinas do dia a dia, necessidade de cuidados e de rever os comportamentos usuais e os posicionamentos táticos na vida ${ }^{2}$. Seja por doença e/ou por outros estados que se prolongam, os quadros de cronicidade impõem às pessoas a convivência com uma nova situação que passa a integrar-se à vida com diversas transformações e (re)arranjos, tanto no plano subjetivo quanto objetivo ${ }^{3}$, seja no âmbito da família, lazer, trabalho etc., requerendo desde o autocuidado até o cuidado como responsabilidade coletiva de bem-estar provido por políticas públicas.

0 trabalho docente, em sua complexidade e ambivalência, abrange aspectos formais e informais, é determinado e contingente, dotado de certa flexibilidade, mas também de controle; pauta-se na atividade de pessoas que trabalham sempre imprimindo sentido ao que fazem, numa interação constante com alunos, colegas, pais, dirigentes da instituição de ensino etc ${ }^{4}$. A escolha em abordar a cronicidade em docentes do ensino 
superior decorreu da percepção pessoal da primeira autora, enfermeira do trabalho em uma instituição de ensino, de que, em meio à responsabilidade de ensinar centenas de alunos, os docentes vivenciam em seu cotidiano sofrimento e prazer no trabalho, de modo que essa dualidade pode estar imbricada com a vivência da condição crônica. Assim, objetivou-se identificar e descrever as características dos estudos científicos nacionais e internacionais sobre a cronicidade em docentes do ensino superior.

\section{METODOLOGIA}

Trata-se de estudo descritivo de revisão integrativa da literatura, partindo da pergunta norteadora pelo mnemônico PICo, sendo seus elementos descritos como $\mathrm{P}=$ População, $\mathrm{I}=$ Fenômeno de Interesse e Co $=$ Contexto, os quais dizem sobre o foco, o escopo e a aplicabilidade da revisão de literatura ${ }^{5}$. Assim, $P$ é representado pelos docentes universitários, I pelas condições crônicas e Co pela universidade, resultando na questão de pesquisa: Como se caracterizam os estudos que abordam condições crônicas em docentes do ensino superior, no cenário nacional e internacional, na atualidade?

Em consonância com a questão de pesquisa, os termos de busca também seguiram a estratégia PICo, mesclando termos dos Descritores em Ciências da Saúde (DeCS/MeSH) e palavras-chave. As publicações foram capturadas no Portal Regional da Biblioteca Virtual de Saúde (BVS) abrangendo todas as suas bases de dados e por meio de buscas diretamente nas bases de dados Scientific Electronic Library 0nline (SciELO), Web of Science (WoS) e Cumulative Index to Nursing and Allied Health Literature (CINAHL), as duas últimas de acesso restrito. A busca eletrônica ocorreu entre dezembro de 2020 e janeiro de 2021, incluindose textos completos disponíveis em periódicos indexados, utilizando-se os filtros de período (2015 a 2020), bem como de idioma (inglês, português e espanhol).

A estratégia de busca está apresentada na Figura 1.
Fiqura 1. Estratéqia de busca, 2021

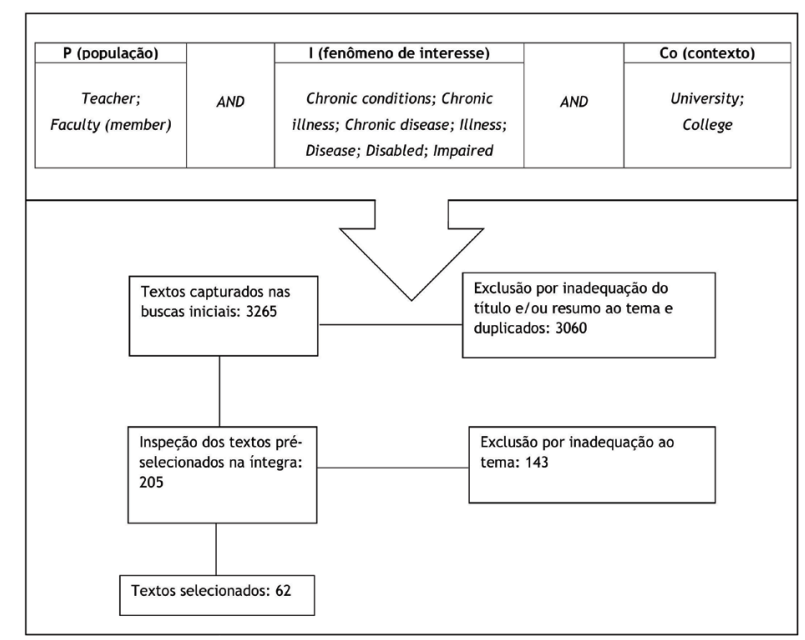

Fonte: Elaboração própria.

Destaca-se que se utilizou a combinação dos termos University e College pelo operador booleano OR na BVS e na base SciELO; já nas bases WoS e CINAHL, optou-se pela busca individual destes termos combinados aos demais componentes $P$ e I da estratégia PICo. 0 descritor Faculty foi usado nas bases SciELO e CINAHL; Faculty member na BVS e WoS, ambos combinados aos elementos P e I da estratégia PICo. Tais combinações visavam evitar a captura excessiva e inespecífica de textos, o que inviabilizaria a verificação da amostra. Quanto aos recursos buscadores, na BVS foram selecionados "Título, resumo, assunto"; na WoS, utilizouse a modalidade "Tópico"; e na base ScieELO foi selecionado o item "Todos os índices".

Da expressão preliminar que capturou 3265 publicações, procedeu-se à leitura do título e/ou resumo e exclusão de 3060 textos por inadequação ao tema e os duplicados. Esses 205 textos préselecionados foram inspecionados quanto ao seu resumo e conteúdo, resultando na criteriosa exclusão de 143 textos por se tratarem de estudos: a) sobre aspirantes à docência; b) sobre professores de outros níveis de ensino; c) que não mencionavam o nível de atuação dos docentes; d) de validação de instrumentos, cartilhas, questionários e e) de revisões de literatura. Com tal exclusão foram alcançados 62 trabalhos elegíveis para a análise (61 artigos e 1 tese) a partir da leitura na integra e sistematização de cada um em planilha do Microsoft Excel 2010, extraindo-se as informações relevantes para a respectiva categorização, descrição e interpretação. A sistematização, tratamento e análise dos artigos foi realizada por duas 
pesquisadoras, de forma independente.

\section{RESULTADOS E DISCUSSÃO}

Os resultados foram organizados em dois subitens que se ocupam das características gerais das produções (país de origem, ano de publicação, tipo do estudo, referencial teórico e sexo da população estudada) e dos temas centrais e respectivas discussões das publicações, incluindo produções sensíveis ao contexto pandêmico da Covid-19.

\section{CARACTERÍSTICAS GERAIS DAS PUBLICAÇÕES}

Em relação ao país de origem dos estudos, demonstra-se graficamente sua distribuição:

Figura 2. Distribuição de textos por país de publicação, 2021.

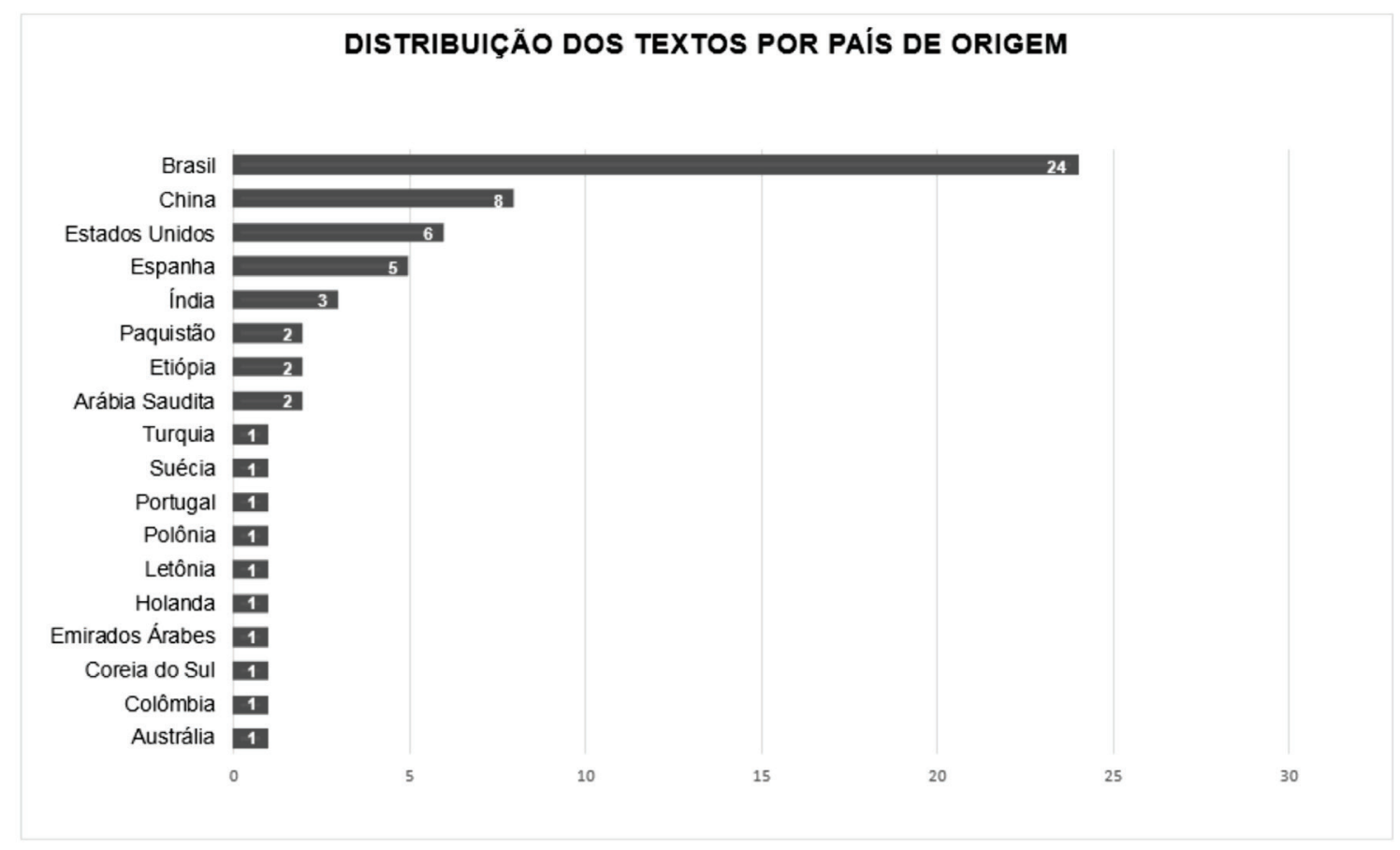

Fonte: Elaboração própria.

Nota-se a predominância de estudos produzidos no Brasil (38\%), seguidos pela China (12\%) e Estados Unidos ( $9 \%)$. No Brasil, a abordagem da saúde dos docentes sofreu um incremento a partir dos anos 90 , na busca por conhecer as consequências da precarização do sistema educacional, diante da ampliação do acesso ao ensino e a intensificação do trabalho docente, decorrentes das reformas nos sistemas de ensino nos âmbitos federal, estadual e municipal, em um contexto de políticas de democratização do ensino visando à equidade social ${ }^{6}$. Tem despertado o interesse de pesquisadores especialmente do campo da saúde do trabalhador, cuja referência central é o processo de trabalho, no qual a articulação dos elementos nele presentes propicia condições geradoras de saúde ou de doença e sofrimento ${ }^{7}$.

A distribuição dos textos quanto ao ano de publicação e ao tipo do estudo é demonstrada a seguir, a partir da Figura 3. 
Figura 3. Distribuição dos textos conforme o ano de publicação e tipo de estudo.

\section{Distribuição dos textos conforme o ano de publicaçãoe tipo de estudo}

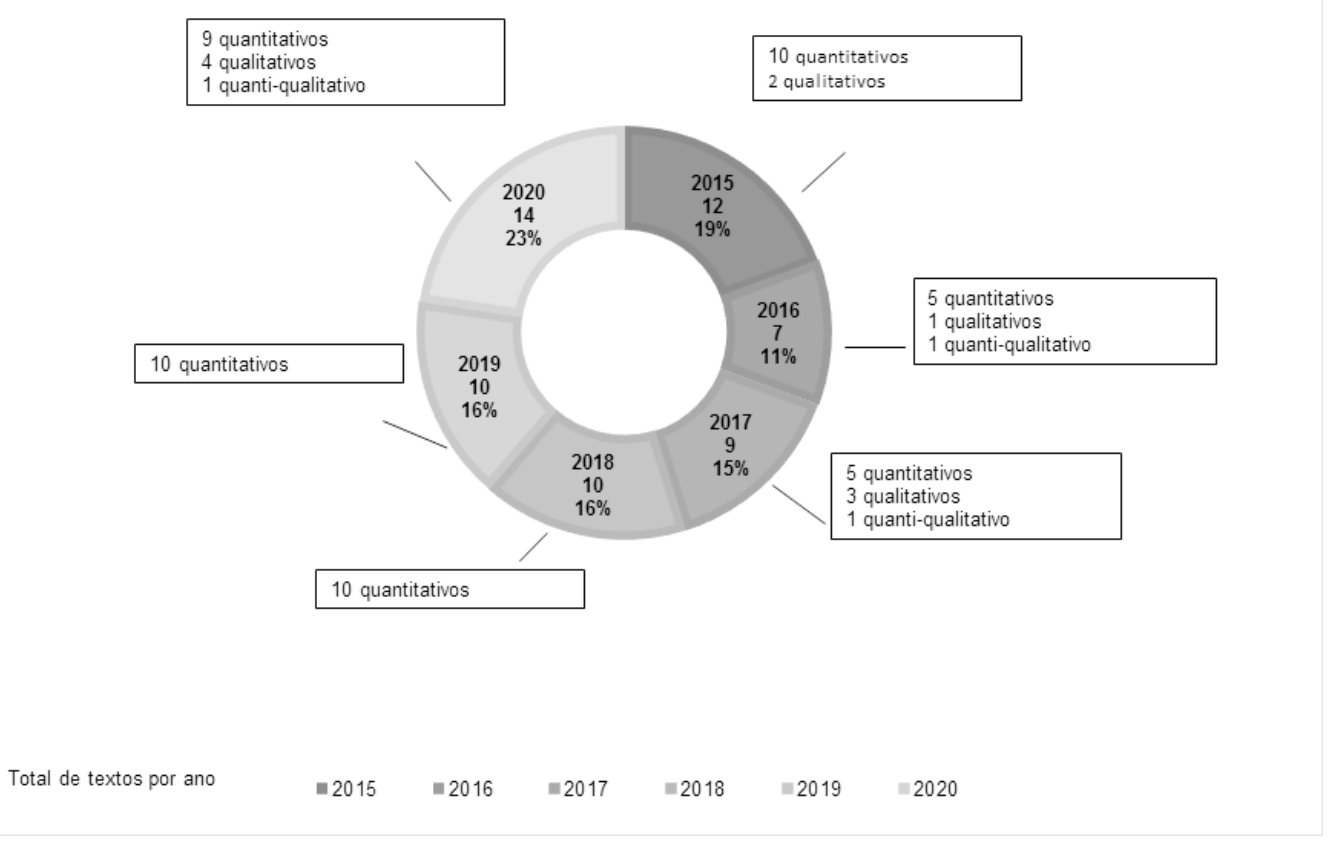

Fonte: Elaboração própria.

Ao todo, 49 (79\%) estudos são do tipo quantitativo, dez (16\%) são qualitativos e três ( $4 \%)$ são quanti-qualitativos. Sendo a maioria das pesquisas quantitativas publicadas no Brasil (17, $27 \%$ ), cabe mencionar que a predominância desse tipo de estudo ocorre desde a década de 90, quando houve demanda inicial por análise da situação da saúde do docente brasileiro, com ênfase na identificação dos principais problemas de saúde e sua relação com as condições do trabalho7.

0ito textos (13\%) explicitaram o referencial teórico adotado, sendo a psicodinâmica do trabalho 0 mais mencionado, constando em quatro publicações. Considerando que estes quatro estudos são brasileiros, é importante mencionar que a produção nacional com esse referencial iniciou na década de 80 , firmando-se na discussão das consequências do trabalho sobre a saúde psíquica dos trabalhadores, investigando o sofrimento e o prazer no trabalho, bem como a elaboração do equilíbrio psíquico diante do desafio de se manterem saudáveis, mesmo em ambientes hostis de trabalho ${ }^{8}$. Dentre os demais textos, um assumiu o construto de inteligência emocional de Mayer e Salovey, um adotou o modelo de crenças da psicologia da saúde, um seguiu o conceito teórico de "estigma" de Erwing Goffman, expoente do Interacionismo Simbólico, e um se apropriou do referencial marxista da corrente Materialista que teve/tem grande influência nos estudos brasileiros sobre o tema que envolve trabalho.

Quanto ao sexo da população estudada, 29 $(46 \%)$ textos decorreram de estudos com maioria de pessoas do segmento feminino e 14 (22\%) com maioria do segmento masculino; sete (11\%) abordaram exclusivamente docentes do segmento feminino e um ( $1 \%$ ) exclusivamente do segmento masculino. Três (5\%) textos apresentaram paridade de sexo e um (1\%) dividiu os segmentos por área de atuação docente, sendo que as áreas de humanas, exatas e agrárias tinham maioria masculina e a área da saúde era composta majoritariamente pelo segmento feminino. Sete $(11 \%)$ publicações não especificaram o sexo dos docentes da amostra.

\section{TEMAS CENTRAIS E DISCUSSÕES GERAIS DAS PUBLICAÇÕES}

Os temas centrais trabalhados nos estudos foram classificados em três categorias, a saber: Avaliação das Condições de Saúde (ACS); Relações entre Trabalho e Subjetividade (RTS) e Conhecimento em Saúde (CS), conforme distribuição na Figura 4. 
Figura 4. Distribuição dos temas conforme o ano das publicações que abordam cronicidade em docentes, 2021.

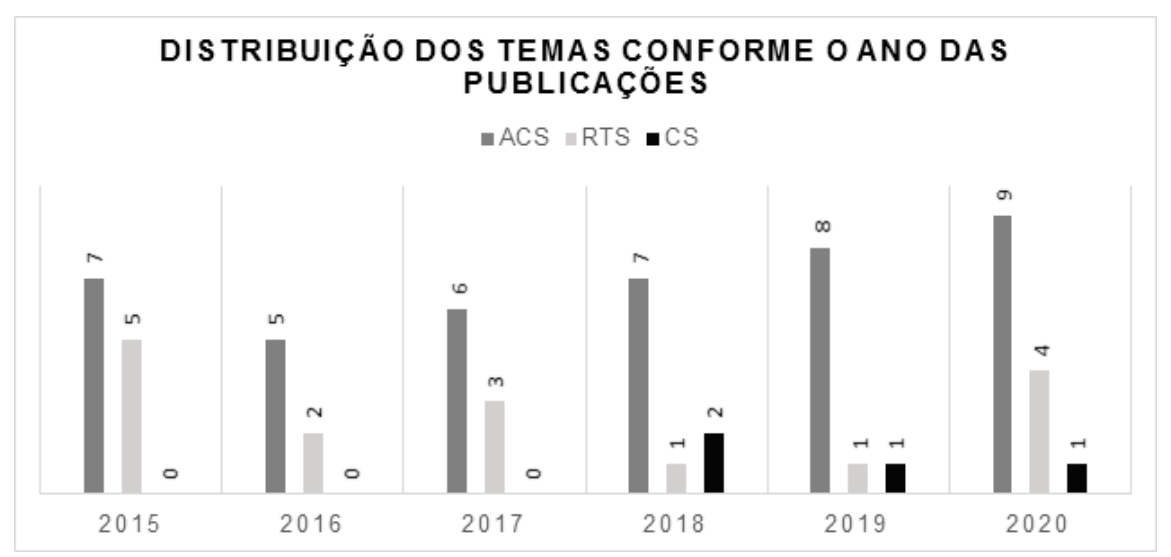

Fonte: Elaboração própria.

0 tema ACS, que predominou dentre os demais $(42,68 \%)$, apresenta um panorama da condição de saúde mental ou física dos docentes universitários, identificando fatores críticos do contexto laboral, efeitos de estresse e esgotamento mental, capacidade de regulação emocional, impactos na qualidade de vida, aspectos nutricionais e riscos para a saúde física. Por sua recorrência, nota-se certa preocupação científica sobre o tema ACS. Considerando que, muitas vezes, os pesquisadores trazem elementos das realidades que eles mesmos vivenciam no cotidiano da universidade, esse achado reforça a necessidade de que a saúde dos docentes universitários seja vista e considerada no que tange às políticas públicas de amparo ao professorado.

Os textos cujo tema central foram as RTS $(16,26 \%)$ enfocam a percepção do trabalho docente, as implicações da precarização do trabalho na saúde do professorado, bem como a associação entre transtornos mentais comuns e estressores no trabalho entre professores. Já o tema CS (4,6\%) consta em estudos que exploram a percepção dos professores em relação ao próprio corpo no ambiente de trabalho, conhecimentos sobre saúde, higiene vocal e terapias nutricionais.

Destacam-se, a partir dos textos arrolados nesta revisão, algumas discussões que envolvem cronicidade em docentes universitários no âmbito nacional e internacional. Tais discussões serão apresentadas de modo desatrelado aos temas centrais pelos quais os estudos foram classificados, posto que elas os transpassam, exceto pela discussão sobre o contexto pandêmico da doença do novo coronavírus (Covid-19), que ocorreu em textos do tema ACS.

As condições crônicas vivenciadas por docentes universitários mais frequentemente abordadas nas publicações estão distribuídas na Figura 5.

Figura 5. Distribuição das publicações conforme o tipo de condição crônica em docentes universitários, 2021.

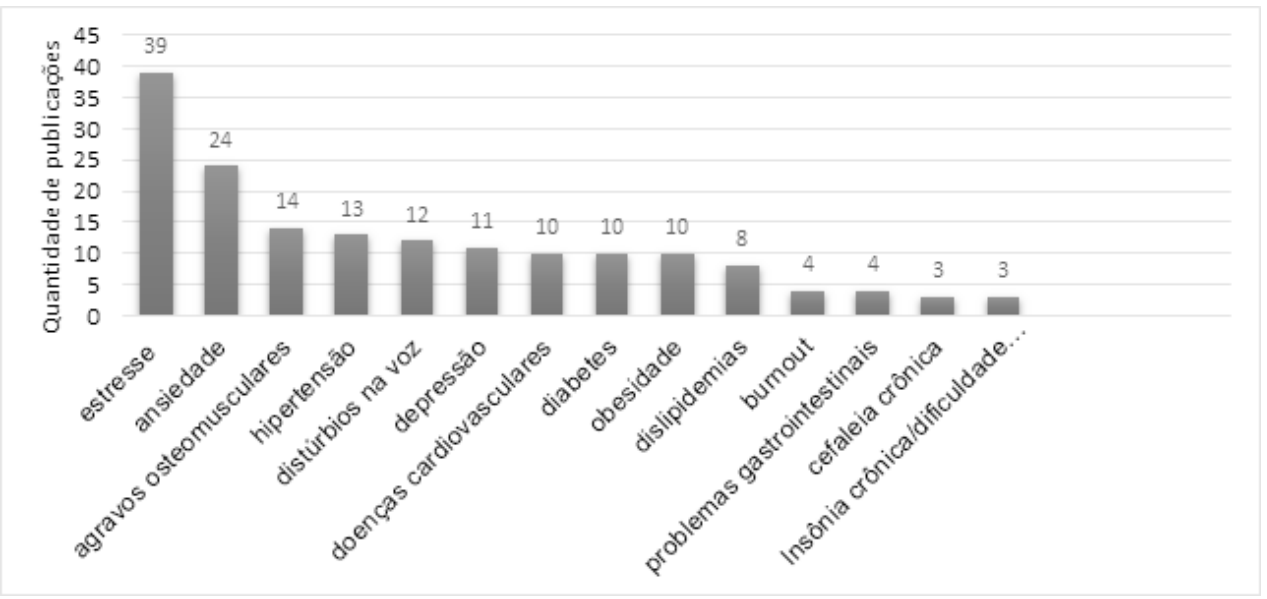

Fonte: Elaboração própria. 
Dentre todos os textos analisados, 16 (26\%) deram relevo às questões emocionais e psíquicas que perpassam o cotidiano do trabalho docente, demonstrando relações importantes entre a satisfação pessoal e o bem-estar no trabalho como principal discussão. 0 estresse é apontado em 39 estudos como relacionado às peculiaridades do trabalho docente no ensino superior, o que caracteriza sua cronicidade, ainda que haja variações decorrentes do contexto.

Estudo brasileiro mencionou que, por ser geradora de estresse, a carreira docente pode estar ligada ao surgimento ou agravamento de outras condições crônicas, tais como dores na coluna, depressão, alteração vocal e hipertensão ${ }^{9}$. De modo semelhante, estudo paquistanês ${ }^{10}$ verificou que professoras universitárias estressadas sofrem de cefaleia e aumento frequente da pressão arterial. Estudo da China mostrou que a falta de financiamento para pesquisas, de descanso rotineiro nos fins de semana ou feriados, de atividades físicas, bem como exigências de titulação acadêmica e pressão para pesquisas científicas foram as principais fontes de estresse dos docentes universitários chineses ${ }^{11}$. Outro estudo chinês ${ }^{12}$ ponderou que o estresse no trabalho também tem efeitos deletérios sobre a confiança e interfere nas interações interpessoais. $\mathrm{Na}$ Índia, pesquisadores perceberam que identificar o estresse possibilita prever alterações na saúde mais eficientemente do que a identificação da satisfação no trabalho ${ }^{13}$.

Dos 62 textos, 35 (56\%) mencionam a associação entre o trabalho docente e variadas condições crônicas, sendo estresse, ansiedade, depressão, problemas na voz e acometimentos osteomusculares as mais expressivas nos estudos brasileiros, dado que vai ao encontro do que foi apontado em estudo anterior ${ }^{7}$. No âmbito internacional, os estudos que abordaram a referida associação trataram das seguintes condições (conforme o país da publicação): China - estresse, depressão, ansiedade, polimorfismos OXTR rs53576 e OXTR rs2268490 (polimorfismos do gene do receptor de ocitocina que moderam a relação entre estresse no trabalho e confiança geral ${ }^{12}$ ); Espanha - estresse e ansiedade, mal-estar psicológico (inclui estresse, depressão e raiva) e desgaste vocal; Colômbia estresse e ansiedade; Índia - estresse, ansiedade e burnout; Letônia - distúrbios vocais; Holanda - estresse, fadiga cognitiva, emocional e física; Paquistão - estresse, hipertensão e cefaleia;
Polônia - estresse, cardiomiopatia de Takotsubo; Portugal - patologia laríngea; Arábia Saudita desordens musculoesqueléticas, estresse; Suécia - problemas de saúde; e Coreia do Sul - burnout.

0ito estudos brasileiros abordaram a intensificação do trabalho docente universitário, decorrente principalmente da expansão das universidades federais e suas demandas de interiorização no Brasil, que proporcionaram acesso à educação sem se adequar às necessidades dos docentes ${ }^{14}$. A fim de cumprir metas da organização laboral, docentes universitários conciliam atividades de ensino, pesquisa, extensão e atividades administrativas ${ }^{9}$; essa configuração é desfavorável à manutenção de hábitos de vida saudáveis pelos docentes e propícia às atitudes negligentes com o cuidado à própria saúde, acarretando consequências negativas para si e para as instituições ${ }^{15}$. É preciso reconhecer que sofrimentos laborais podem culminar em adoecimento, mas também podem não se manifestar como doenças que requerem medicação e afastamento, presentificando-se em diversos sintomas danosos ao bem-estar do professor universitário ${ }^{16}$, gerando sofrimento silencioso e invisibilizado, mas não menos pernicioso.

Alguns estudos $(9,14 \%)$ discorreram sobre questões de gênero, enfocando geralmente a inserção da mulher no mercado de trabalho e suas consequências. Nos estudos brasileiros, as discussões foram polarizadas entre a possibilidade de ascensão e autonomia econômica feminina e a percepção de que as condições de trabalho são insatisfatórias devido aos danos físicos e emocionais acarretados pela dupla função entre trabalhar como docente e cuidar da família. Semelhantemente, estudo paquistanês apontou que docentes universitárias possuem pouco apoio de colegas, menores salários, perfazem longa jornada e alta carga de trabalho e vivenciam atitudes exigentes de familiares, fatores que desencadeiam estresse, afetando sua saúde física e psicológica ${ }^{10}$. Estudo espanhol ${ }^{17}$, que abordou a desmotivação em professoras e professores, elaborou o retrato do professor universitário desmotivado: jovem solteira, trabalhadora da área social, instável na carreira docente e que leciona há alguns anos. Dentre seus resultados, destacase a vulnerabilidade de docentes universitárias que vivenciam abusos e pressões sexistas, muitas vezes institucionalizados, forçando-as a abandonar 
planos pessoais como o da maternidade ao negar a concessão de licença-maternidade ${ }^{17}$.

$\mathrm{Na}$ amostra, 11 (17\%) artigos evidenciaram a correlação entre hábitos de vida e saúde dos docentes universitários, tais como os benefícios da prática regular de exercícios físicos, boa alimentação e sono reparador, da mesma forma que hábitos como o tabagismo e alcoolismo foram apontados como fatores deletérios. Estudo chinês concluiu que a concepção de alimentação saudável deve ser enfatizada para os professores, pois hipertensão, hiperglicemia, insônia e hiperlipidemia foram todas expressivamente associadas aos seus hábitos alimentares não saudáveis ${ }^{18}$. Pesquisadores indianos verificaram que professores universitários carecem de conhecimento sobre nutrição na hipertensão e sugerem urgência em melhorar a conscientização sobre o assunto, destacando o papel dos docentes como importantes divulgadores de conhecimento da sociedade ${ }^{19}$.

Em pesquisas brasileiras, os professores universitários que praticam atividade física apresentaram melhor qualidade de vida e de trabalho e relataram melhor qualidade de sono. Porém, em geral, os estudos evidenciaram baixos níveis de atividade física nesses docentes. 0s professores fisicamente inativos tinham menos atividades de lazer e uma dieta com menor consumo de frutas, verduras e legumes e consumo excessivo de carne com gordura, refrigerante e álcool ${ }^{20}$. Alimentação não saudável associou-se com acidente vascular cerebral; atividade física irregular/sedentarismo com doenças endócrinas/ metabólicas e do aparelho digestivo; obesidade com doenças cardiovasculares/endócrinas/ metabólicas e diabetes; e o tabagismo com doenças osteomusculares ${ }^{21}$. Já o estudo que analisou a qualidade do sono dos docentes universitários constatou que esses são maus dormidores e que há associação entre a má qualidade do sono e os fatores de risco para as doenças cardiovasculares ${ }^{22}$.

No contexto pandêmico da Covid-19, anunciado pela Organização Mundial da Saúde em 11 de março de $2020^{23}$, o avanço exponencial de vidas perdidas pela doença em nível global tem proporcionado um convívio com a morte e o morrer e a busca por meios de superação e adaptação a esta realidade atípica e singular ${ }^{24}$. Cinco estudos quantitativos abarcaram este contexto: três estudaram os efeitos psicológicos em docentes universitários ${ }^{25-27}$, um abarcou as percepções dos docentes sobre a pandemia e sua adesão aos comportamentos de proteção à saúde ${ }^{28}$ e um apontou o impacto da pandemia na educação on-line em uma faculdade de medicina ${ }^{29}$. Nesse último, oriundo de pesquisa na Arábia Saudita ${ }^{29}$, a maioria dos docentes relatou confiança na potência do ensino on-line durante as primeiras semanas da pandemia e que a experiência on-line adquirida neste período poderia integrarse em suas estratégias de ensino. Não obstante, apontam desafios relacionados à comunicação, avaliação do aluno, uso de ferramentas tecnológicas, experiência on-line, gerenciamento de tempo, ansiedade ou estresse relacionados à pandemia e tecnofobia ${ }^{29}$.

A ansiedade entre professores chineses no contexto pandêmico foi foco de estudo que destacou a necessidade de considerar fatores como idade, sexo, escolaridade, localização da instituição de ensino, fontes de informação, níveis de preocupação e de medo e estado de comportamento no gerenciamento da ansiedade ${ }^{25}$. Estudo espanhol ${ }^{26}$ avaliou que cerca de metade dos entrevistados locais apresentou impacto moderado a grave do surto, apresentando sintomas de estresse, ansiedade e depressão, além de altos níveis de preocupação. Estudo norte-americano ${ }^{27}$ verificou que a maioria dos docentes trabalhava em casa, e que sua carga de trabalho aumentou, além da piora no bem-estar geral relacionada ao trabalho ou às mudanças de vida decorrentes da pandemia de Covid-19. Estresse também foi relacionado às finanças pessoais, à manutenção do emprego e da sua própria saúde ou de amigos/família frente ao risco de infecção; além de ser apontado por aqueles cujos filhos estavam em casa em ensino doméstico e por aqueles que cuidam de parentes idosos. A exposição à Covid-19 (diagnóstico próprio ou familiar, ou exposição a alguém possivelmente contaminado) foi associada a maior estresse, ansiedade, depressão e exaustão no trabalho. Mulheres foram mais propensas a relatar ansiedade, exaustão no trabalho e diminuição do bem-estar ${ }^{27}$, evidenciando, assim, diferentes impactos da pandemia balizados pelas desigualdades sociais, no caso, de gênero.

Estudo conduzido nos Emirados Árabes ${ }^{28}$ apontou que participantes com vínculo empregatício, ensino superior completo e aqueles com diagnóstico de doença crônica apresentaram maior probabilidade de aderir aos comportamentos preventivos da Covid-19. Fornecer informações claras, consistentes e focadas em ações simples 
que os indivíduos podem realizar para reduzir o risco de infecção, destacando-se sua eficácia, pode maximizar a adesão, apesar da presença de medo e ansiedade. Pontuou ainda a diferença entre ansiedade patológica e "ansiedade funcional", que ocorre no contexto de preparação para uma ameaça tangivel, sem descartar a possibilidade de que docentes com fatores de risco para doenças mentais possam vivenciar a pandemia como um precipitante para o sofrimento patológico e o desenvolvimento de estratégias de enfrentamento mal adaptativas, destacando o papel fundamental dos profissionais de saúde mental neste contexto ${ }^{28}$.

Vinte e seis (42\%) textos sugerem medidas a serem implementadas na intenção de oferecer algum suporte em âmbito institucional à saúde dos docentes universitários. Dentre elas, destacam-se: criação de políticas institucionais e governamentais relacionadas à promoção da saúde na educação superior; incentivo à prática de atividades físicas pelos docentes; promoção da saúde vocal; melhoria no diálogo entre docentes e gestores a fim de eliminar fatores de risco de adoecimento mental e conquistar melhores condições de trabalho; aconselhamento psicológico e cursos de gerenciamento de estresse, inclusive considerando o contexto pandêmico; adequações institucionais, legais, curriculares e sociais para aprimorar o processo ensino-aprendizagem e a qualidade de vida dos docentes.

\section{CONCLUSÃO}

As pesquisas têm apresentado discussões ampliadas sobre cronicidade em docentes universitários, que extrapolam o olhar biomédico focado apenas na patologia e seus fatores de risco, considerando também o seu contexto de trabalho e fatores históricos relacionados, tais como a intensificação do trabalho docente no contexto brasileiro. Incentiva-se, porém, a ampliação do desenvolvimento da temática por meio de estudos qualitativos em âmbito nacional e internacional, posto que tal abordagem possibilita maior aproximação aos meandros da experiência cotidiana da condição crônica vivida por docentes universitários, considerando a vida nos seus diversos espaços e modos de acontecer.

Destaca-se o predomínio do estresse no cotidiano do docente universitário, constituindose como uma condição crônica marcada por pressão emocional durante período prolongado. Ainda que não seja reconhecido como doença, favorece o surgimento/agravamento/manutenção de doenças, inclusive cronificadas, sendo, por vezes, banalizado/invisibilizado. Sugere-se a ampliação dos estudos sobre o estresse que forneçam mais informações sobre como ele é vivido no cotidiano do docente universitário.

Para potencializar a captura de estudos sobre a cronicidade em docentes universitários com deficiências, empregou-se os termos disabled e impaired, mas nenhuma publicação foi incluída, pois os estudos capturados tratavam sobre deficiências em alunos e o docente no processo de seu acolhimento. Ainda que essa seja uma constatação não analisada e considerando a não captura de estudos sobre deficiências nos docentes universitários, recomenda-se o refinamento das buscas e a realização de estudos específicos que considerem a temática em questão.

Com relação às revisões de literatura anteriores, o presente estudo avançou abordando a docência sob a ótica da cronicidade e expandindo o olhar para as produções em âmbito internacional, a fim de conhecer o que se tem produzido sobre a temática e traçando paralelos com o que se identificou nos estudos nacionais. Avança, também, ao abarcar publicações produzidas no contexto pandêmico, evidenciando que, no que tange às condições crônicas em docentes universitários, há necessidade de condução de mais estudos sobre as implicações das medidas de mitigação da disseminação do novo coronavírus que impactaram diretamente no trabalho docente. Há de se considerar que, nessa população, outras situações de cronicidade, assim como as desigualdades no contexto social e profissional em que elas transcorrem, podem ser agravadas e amplificadas pela pandemia, que vem se mostrando cronificada.

Dentre as limitações do estudo, destacam-se a ausência de análise da qualidade dos textos e a inclusão de docentes de diferentes níveis de ensino, envolvendo graduação e pós-graduação. Contudo, os resultados identificados permitiram destacar lacunas do conhecimento e apontar perspectivas para a discussão sobre a cronicidade como parte da vida do professor universitário.

\section{CONTRIBUIÇÃO DAS AUTORAS}

Karla Beatriz Barros de Almeida contribuiu 
no delineamento, revisão da literatura, análise e redação do estudo. Juliana Soares Androlage contribuiu na revisão de literatura e revisão crítica do estudo. Reni Barsaglini contribuiu no delineamento e revisão crítica do estudo.

\section{REFERÊNCIAS}

1. Mendes EV. Entrevista: A abordagem das condições crônicas pelo Sistema Único de Saúde. Ciên Saúde Colet [serial on the internet]. 2018 [cited 2021 Feb 08];23(2):431-35. Available from: https://www.scielo.br/pdf/csc/v23n2/1413-8123csc-23-02-0431.pdf

2. Herzlich C. Saúde e doença no início do século XXI: entre a experiência privada e a esfera pública. Physis. 2004;14(2):383-94.

3. Barsaglini RA. Repercussões dos adoecimentos crônicos nos estudos de experiência: tipos, momentos e mediadores. Oficina do CES. $2019 ; 1(452): 1-27$.

4. Tardif M, Lessard C. 0 trabalho docente hoje: elementos para um quadro de análise. In: Tardif $M$, Lessard $C$, editores. 0 trabalho docente: elementos para uma teoria da docência como profissão de interações humanas. 5. ed. Petrópolis: Vozes; 2009.

5. The Joanna Briggs Institute. Joanna Briggs Institute Reviewers' Manual: 2014 edition/ Supplement. South Australia: The Joanna Briggs Institute; 2014.

6. Assunção $A A$, Oliveira DA. Intensificação do trabalho e saúde dos professores. Educ Soc. $2009 ; 30(107): 349-72$.

7. Araújo TM, Pinho OS, Masson MLV. Trabalho e saúde de professoras e professores no Brasil: reflexões sobre trajetórias das investigações, avanços e desafios. Cad Saúde Pública. 2019;35(Suppl 1).

8. Merlo, ARC, Mendes, AMB. Perspectivas do uso da psicodinâmica do trabalho no Brasil: teoria, pesquisa e ação. Cad psicol soc. 2009;12(2):14156.

9. Soares MB, Mafra SCT, Faria ER. Fatores associados à percepção de estresse em docentes universitários em uma instituição pública federal. Rev Bras Med Trab [serial on the internet]. 2019 [cited 2021 Feb 10];17(1):90-8. Available from: https://www.rbmt.org.br/details/425/pt-BR/ fatores-associados-a-percepcao-de-estresse-emdocentes-universitarios-em-uma-instituicaopublica-federal

10. Mushtaq M. Stress, headache, high blood pressure and self-esteem in working women. Pak
Heart ] [serial on the internet]. 2018 [cited 2021 Feb 12];51(02):166-71. Available from: http:// www.pkheartjournal.com/index.php/pkheart/ article/view/1498

11. Li W, Kou C. Prevalence and correlates of psychological stress among teachers at a national key comprehensive university in China. Int $\mathrm{J}$ 0ccup Environ Health [serial on the internet]. 2018 [cited 2021 Feb 10];24(1-2):7-16. Available from: $\quad$ https://www.tandfonline.com/doi/ full/10.1080/10773525.2018.1500803

12. Fang $Y, L i Z$, Wu $S$, Wang $C$, Dong $Y$, He $S$. oxytocin receptor gene polymorphisms moderate the relationship between job stress and general trust in Chinese Han university teachers. J Affect Disord [serial on the internet]. 2020 [cited 2021 Feb 12];260:18-23. Available from: https:// www.sciencedirect.com/science/article/abs/pii/ $\underline{\text { S0165032719310390 }}$

13. Rana A, Soodan V. Effect of occupational and personal stress on job satisfaction, burnout, and health: A cross-sectional analysis of college teachers in Punjab, India. Indian $\mathrm{J}$ 0ccup Environ Med [serial on the internet]. 2019 [cited $2021 \mathrm{Feb}$ 12];23:133-40. Available from: https://www.ncbi. nlm.nih.gov/pmc/articles/PMC6941331/

14. Tundis AGO, Monteiro JK. Ensino superior e adoecimento docente: um estudo em uma universidade pública. Psic. da Ed. [serial on the internet]. 2018 [cited 2021 Feb 05];46(1):1-10. Available from: https://revistas.pucsp.br/index. $\mathrm{php/psicoeduca/article/view/39139}$

15. D'Oliveira CAFB, Souza NVD0, Varella TCMML, Almeida PF. Configurações do mundo do trabalho e o processo saúde-doença dos trabalhadores docentes de enfermagem. Rev enferm UERJ. 2020;28:e33123. doi: http://dx.doi.org/10.12957/reuerj.2020.33123

16. Ruza, FM, Silva, EP. As transformações produtivas na pós-graduação: o prazer no trabalho docente está suspenso? Rev Subj. 2016;16(1):91-103. doi: http://dx.doi.org/10.5 $\underline{020 / 23590777.16 .1 .91-103}$

17. Jimeno AP, López SC. La desmotivación del profesorado universitario y su relación con variables sociodemográficas, laborales y de personalidade. Apuntes de Psicología [serial on the internet]. 2015 [cited 2021 Feb 13];33(1):516. Available from: http://apuntesdepsicolog ia.es/index.php/revista/article/view/557

18. Zheng C, Shi C, Bai J, Shao W, Zhang C, Bu J. Large-scale investigation on healthy status of teachers in Jiangsu Province, China. Progress in Nutrition [serial on the internet]. 2019 [cited 2021 Feb 13];21(2):294-98. Available from: https://mattioli1885journals.com/index .php/progressinnutrition/article/view/6773 
19. Suchitra MR, Vadivel S, Balasubramanian M, Parthasarathy S. A study of awareness about "Nutrition in Hypertension" among college teachers in a semi urban town of South India. AJPRHC [serial on the internet]. 2018 [cited 2021 Feb 13];10(1):30-35. Available from: http:// www.informaticsjournals.com/index.php/ajprhc/ article/view/18902

20. Lira BA, Souza P, Gomes DJ0, Maniçoba ACM, Monteiro LZ. Inatividade física e fatores de risco para doenças crônicas em professores universitários. Conscientiae Saúde (Online) [serial on the internet]. 2018 [cited 2021 Feb 13];17(4):45462. Available from: https://docs.bvsalud .org/biblioref/2019/04/987380/8748-572531pb. pdf\#: : text=Sobre\%200s\%20fatores\%20de $\% 20$ risco,classificados $\% 20 \mathrm{como \%} 20$ insuficiente $\% 2 \mathrm{D} \% 20 \mathrm{mente} \% 20$ ativos.

21. Setto JM, Bonolo PF, Franceschini SCC. Relationship between health behaviors and self-reported diseases by public employees. Fisioter Mov [serial on the internet]. 2016 [cited https://www.scielo.br/scielo.php?script=sci a rttext\&pid $=$ S0103-51502016000300477

22. Silva KL, Marques ACML, Aragão AV, Gonçalves AV, Feitosa ANA, Araújo WA, et al. Fatores de risco para as doenças cardiovasculares e qualidade do sono. Rev enferm UFPE [serial on the internet]. 2018 [cited 2021 Feb 05];12(10):2573-82. Available from: https://periodicos.ufpe.br/revistas/ revistaenfermagem/article/view/237489/30146

23. Organização Pan-Americana de Saúde. OMS afirma que Covid-19 é agora caracterizada como pandemia - 2020 [home-page on the Internet]. [cited 2021 Feb 03]. Available from: https:// www.paho.org/bra/index.php?option=com content\&view =article \&id $=6120: 0 \mathrm{~ms}$-afirmaque-covid-19-e-agora-caracterizada-comopandemia\&Itemid $=812$

24. Oliveira EN. "Primeiro tivemos que morrer": saúde mental em tempos de covid-19. Sanare (Sobral, Online) [serial on the internet]. 2020 [cited 2021 Feb 05];19(0):05-06. Available from: https://sanare.emnuvens.com.br/sanare/article/ view/1469

25. Li Q, Miao Y, Zeng X, Tarimo CS, Wu C, Wu J. Prevalence and factors for anxiety during the coronavirus disease 2019 (COVID-19) epidemic among the teachers in China. J Affect Disord [serial on the internet]. 2020 [cited 2021 Feb 08];277:15358. Available from: https://www.sciencedirect. com/science/article/abs/pii/S0165032720326136

26. Gonzalez P0, Gomez AP, Irurtia MJ, Garcia RL. Psychological effects of the COVID-19 outbreak and lockdown among students and workers of a Spanish 2021 Feb 15];29(3):477-485. Available from:

university. Psychiatry Research [serial on the internet]. 2020 [cited 2021 Feb 13];290(8):11308. Available from: https://pubmed.ncbi.nlm.nih. gov/32450409/

27. Evanoff BA, Strickland JR, Dale AM, Hayibor L, Page E, Duncan JG, et al. Work-related and personal factors associated with mental well-being during the COVID-19 response: survey of health care and other workers. J Med Internet Res [serial on the internet]. 2020 [cited 2021 Feb 07];22(8):e21366. Available from: https://www.ncbi.nlm.nih.gov/pmc/articles/ PMC 7470175/?report=printable

28. Vally Z. Public perceptions, anxiety and the perceived efficacy of health-protective behaviours to mitigate the spread of the SARS-Cov-2/COVID-19 pandemic. Public Health [serial on the internet]. 2020 [cited 2021 Feb 18];187:67-73. Available from: https://www.sciencedirect.com/science/ article/abs/pii/S0033350620303577

29. Rajab MH, Gazal AM, Alkattan K. Challenges to online medical education during the COVID-19 pandemic. Cureus [serial on the internet]. 2020 [cited 2021 Feb 11];12(7):e8966. Available from: https://www.cureus.com/articles/30131challenges-to-online-medical-education-duringthe-covid-19-pandemic
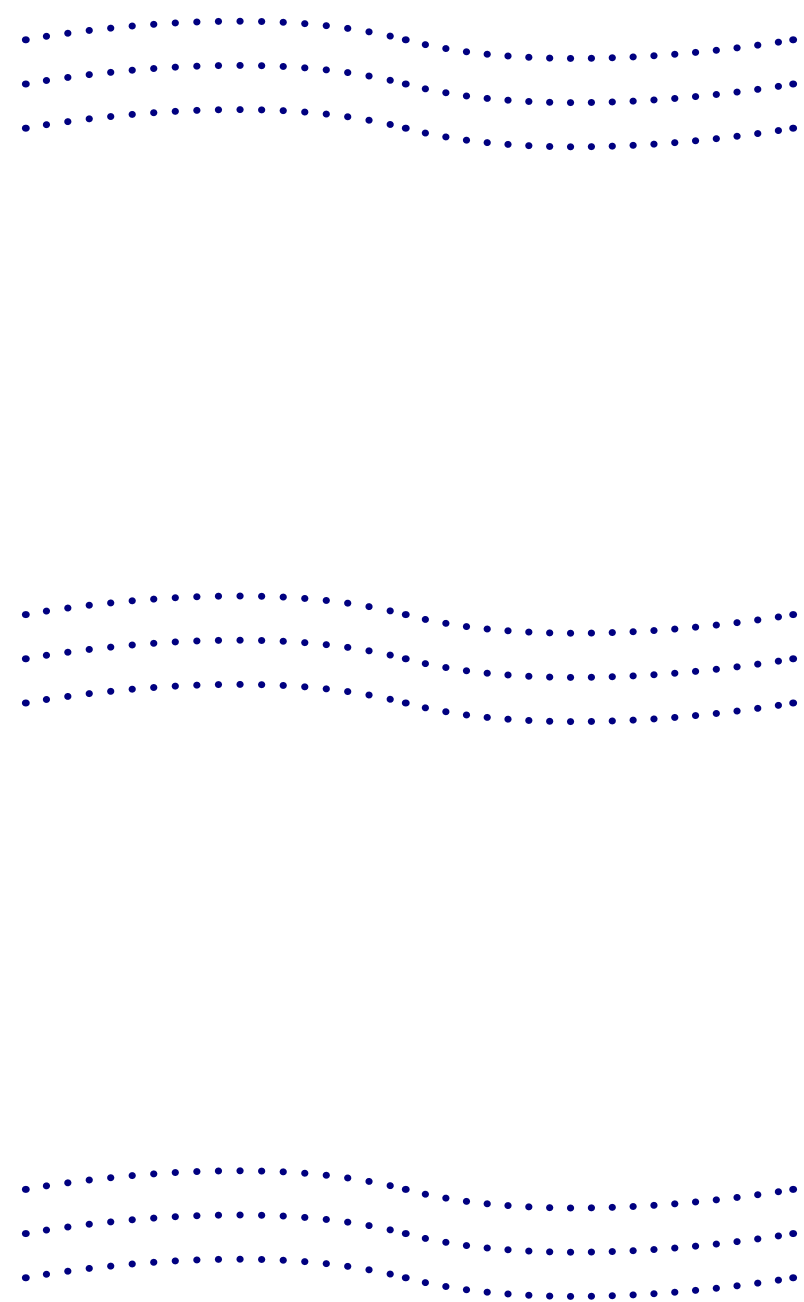

72 - SANARE (Sobral, Online). 2021 Jan-Jun;20(1):62-72 